2. Seymour Ginsburg, Real-valued functions on partially ordered sets, Proc. Amer. Math. Soc. vol. 4 (1953) pp. 356-359.

3. John Tukey, Convergence and uniformity in topology, Princeton, 1940.

Princeton University and

STANFORD UNIVERSITY

\title{
THE ZEROS OF CERTAIN SINE-LIKE INTEGRALS
}

A. E. LIVINGSTON AND LEE LORCH

We establish here the monotonic character of the zeros (modulo 1) of

$$
\int_{x}^{\infty} \frac{f(t)}{t} d t, \quad x>0,
$$

where $f(t)$ satisfies the conditions

(C1). $f(t) \geqq 0$ for $0 \leqq t<1$;

(C2). $f(t) \not \equiv 0$ on any subinterval of $0 \leqq t<1$;

(C3). $f(t+n)=(-1)^{n} f(t)$ for $n=1,2,3, \cdots$;

(C4). $f(t) / t$ is Lebesgue integrable on $0 \leqq t \leqq 1$.

It is clear that these conditions imply that the integral (1) has precisely one zero, say $z_{n}$, in the interval $n<x<n+1$.

Let $C$ be defined (uniquely) by the conditions

$$
2 \int_{0}^{C} f(t) d t=\int_{0}^{1} f(t) d t, \quad 0<C<1 .
$$

Now,

(A) $z_{n}-n \geqq C$ for $n=0,1,2, \cdots$,

(B) $z_{n}-n \rightarrow C$ as $n \rightarrow \infty$,

as was shown in [2], even more generally, with the factor $1 / t$ of $f(t)$ in (1) replaced by a function denoted there by $g(t)$ of which $1 / t$ is a special case. When $f(t)=\sin \pi t$, the sequence $\left\{z_{n}-n\right\}_{0}^{\infty}$ is decreasing, as Harry Pollard has shown, and I. I. Hirschman has observed that Pollard's proof applies equally well to the zeros of

$$
\int_{x}^{\infty} g(t) \sin \pi t d t
$$

Presented to the Society June 18, 1955; received by the editors December 13 . 1954 and, in revised form, October 11, 1955. 
where $g(t)$ is completely monotonic in $0<t<\infty$ [3, pp. 409-411]. (Here $g(t)$ has a meaning different from the one in [2].)

We prove here the following result:

Let $f(t)$ satisfy the conditions $(\mathrm{C} 1)-(\mathrm{C} 4)$, and denote by $z_{n}$ the unique zero of (1) in the interval $n<x<n+1, n=0,1,2, \ldots$. Then $z_{n}-n \downarrow C$, where $C$ is defined by (2).

In replacing $\sin \pi t$ in $\operatorname{si}(\pi x)$ by a more general function the above result extends Theorem 3.2 of [3] in one direction, while Hirschman's observation concerning (3) generalizes that theorem in another fashion by replacing $1 / t$ in $\operatorname{si}(\pi x)$ by an arbitrary completely monotonic function $g(t)$.

In view of $(\mathrm{B})$, it is only the monotonicity of the sequence $\left\{z_{n}-n\right\}_{0}^{\infty}$ that need be established. The formula

$$
\int_{x}^{\infty} \frac{f(t)}{t} d t=\int_{x}^{1+x} f(t) \sum_{i=0}^{\infty} \frac{(-1)^{i}}{t+i} d t
$$

is obtained by writing (1) in the natural way as a sum of integrals over subintervals of $[x, \infty]$ of length one, making a linear change of variable $t=t^{\prime}+i$ in each of these, and then interchanging the order of summation and integration. The function represented by the infinite series in (4) is denoted customarily $[1$, p. $20(6)]$ by $G(t) / 2$. With this notation, we have

$$
\int_{z_{n}}^{1+z_{n}} f(t) G(t) d t=0
$$

Suppose that $G_{0}(t)$ is a non-negative increasing function of $t$ for $0<t<\infty$. By the second mean-value theorem, (A), and (5),

$$
(-1)^{n} \int_{z_{n}}^{1+z_{n}} f(t) G(t) G_{0}(t) d t=(-1)^{n} G_{0}\left(1+z_{n}\right) \int_{\xi_{n}}^{1+z_{n}} f(t) G(t) d t \leqq 0,
$$

where $z_{n}<\xi_{n}<z_{n}+1$. Thus, if there is an $\alpha, n+C \leqq \alpha<n+1$, for which

$$
\int_{\alpha}^{\alpha+1} f(t) G(t) G_{0}(t) d t=0
$$

then, necessarily, $\alpha \leqq z_{n}$. Since

$$
\begin{aligned}
0 & =\int_{z_{n+1}-1}^{1+z_{n+1}} f(t) G(t) d t=-\int_{-l+z_{n+1}}^{z_{n+1}} f(t) G(t+1) d t \\
& =-\int_{-l+z_{n+1}}^{z_{n+1}} f(t) G(t) \frac{G(t+1)}{G(t)} d t
\end{aligned}
$$


it follows by this argument that $z_{n+1}-(n+1) \leqq z_{n}-n$, provided only that $G(t+1) / G(t)$ is increasing for $0<t<\infty$. We show now that this is the case.

Recalling the definition of $G(t)$, it is easy to verify that $G(t)$ $+G(t+1)=2 / t[1$, p. $20(7)]$. Thus, we may accomplish our aim by showing that $t G(t)$ is decreasing for $t>0$.

FIRST PROOF. (This was suggested in conversation with M. Riesz.) We have $[1$, p. 20(2)]

$$
t G(t)=2 t \int_{0}^{1} \frac{r^{t-1}}{1+r} d r .
$$

Integrating by parts,

$$
t G(t)=1+2 \int_{0}^{1} \frac{r^{t}}{(1+r)^{2}} d r
$$

from which the desired result follows immediately.

REMARKS. On successive differentiation, (6) shows that $t G(t)$ is completely monotonic, $0<t<\infty$. This is true also of $t^{\delta} G(t)$ for any $\delta<1$, since $t^{\delta} G(t)$ can be written as the product of the two completely monotonic functions $1 / t^{1-\delta}, \delta<1$, and $t G(t)$. [That the product of two completely monotonic functions is also completely monotonic follows at once from the successive differentiation of that product by Leibniz's rule.]

Moreover, the restriction $\delta \leqq 1$ cannot be removed if the function $t^{\delta} G(t)$ is to be completely monotonic, $0<t<\infty$, since $t^{\delta} G(t)$ increases rather than decreases (as required for complete monotonicity), at least for some positive interval of values of $t$, for any $\delta>1$.

To see this, let $\delta=1+\epsilon, \epsilon>0$. Then

$$
\begin{aligned}
{\left[t^{1+e} G(t)\right]^{\prime} } & =t^{\epsilon}\left[(1+\epsilon) G(t)+t G^{\prime}(t)\right] \\
& =2 t^{\epsilon} \sum_{n=0}^{\infty} \frac{(-1)^{n}}{t+n}\left\{1+\epsilon-\frac{t}{t+n}\right\},
\end{aligned}
$$

where the infinite series representation is found directly or is taken from $[1$, p. $20(6)$, p. $45(10)]$.

This series is an alternating series whose first term is positive. The series itself will be shown to be positive for certain values of $t$, and the function $t^{1+\epsilon} G(t)$ to be increasing there, once we show that the terms of that series are monotonically decreasing for those values of $t$. Now, we observe that this is the case if 


$$
t\left\{\frac{2 t+2 n+1}{(t+n+1)(t+n)}\right\} \leqq 1+\epsilon, \quad n=0,1,2, \cdots .
$$

We note that the expression in braces decreases as $n$ increases. Thus, the left member of ( 7$)$ is greatest when $n=0$, i.e., its maximum is $(2 t+1) /(t+1)$. But this maximum is $\leqq 1+\epsilon$ when $t^{-1} \geqq(1-\epsilon) / \epsilon$ and so we have shown that $t^{1+\epsilon} G(t)$ cannot be completely monotonic, $0<t<\infty$, for any $\epsilon>0$ whatever.

Some interest may attach to the above observation, since $G(t)$ is a standard "special function" and can be defined in terms of $\psi(t)$, the logarithmic derivative of the gamma function. Doing so, we can express these results as follows:

The function $t^{8}[\psi(t+1 / 2)-\psi(t)]$ is completely monotonic, $0<t<\infty$, if and only if $\delta \leqq 1$.

If $1<\delta<2$, the function increases for $0<t \leqq(\delta-1) /(2-\delta)$. if $\delta \geqq 2$, it increases for all $t>0$.

In case $\delta=1$, this shows $[1$, p. 20 (6)] that the hypergeometric function ${ }_{2} F_{1}(1, t ; 1+t ;-1)$ is also completely monotonic, $0<t<\infty$.

SECOND PROOF. To show alternatively that $t G(t)$ decreases as $t$ increases, $0<t<\infty$, we put $2 t=1 / s$ and use $[1$, p. $20(6)]$, whence

$$
2 t G(2 t)=2 \frac{1}{s} \sum_{n=0}^{\infty} \frac{(-1)^{n}}{1 / s+n}=2 \sum_{n=0}^{\infty} \frac{(-1)^{n}}{1+n s}=2 \int_{0}^{1} \frac{d r}{1+r^{s}} .
$$

The last expression clearly decreases from 2 to 1 as $s$ decreases from $\infty$ to 0 , that is, as $t$ increases from 0 to $\infty$.

\section{REFERENCES}

1. A. Erdélyi et al, Higher transcendental functions (based, in part, on notes left by Harry Bateman), vol. 1, New York, 1953.

2. A. E. Livingston, The zeros of a certain class of indefinite integrals, Proc. Amer. Math. Soc. vol. 5 (1954) pp. 296-300.

3. - Some Hausdorf means which exhibit the Gibbs' phenomenon, Pacific J. Math. vol. 3 (1953) pp. 407-415.

UNIVERSITY OF WASHINGTON AND Philander Smith College 